\title{
DEVELOPMENT OF A THERMOMECHANICAL MODEL FOR PREDICTION OF RESIDUAL STRESS DURING LASER POWDER- BED FUSION: EVALUATION OF INHERENT STRAIN APPROACH
}

\author{
JEAN-PHILIPPE MARCOTTE ${ }^{1}$, KALONJI KABANEMI' ${ }^{1}$, MARJAN MOLAVI- \\ ZARANDI ${ }^{1}$, FLORIN ILINCA ${ }^{1}$, JEAN-SEBASTIEN CAGNONE ${ }^{1}$ \\ ${ }^{1}$ National Research Council Canada (NRC), 75 Boulevard de Mortagne, Boucherville, QC J4B 6Y4 \\ Jean-Philippe.Marcotte@cnrc-nrc.gc.ca \\ KalonjiKabaa.Kabanemi@cnrc-nrc.gc.ca \\ Marjan.Molavi-Zarandi@enrc-nrc.gc.ca \\ Florin.Ilinca@cnrc-nrc.gc.ca
}

Key words: Laser Powder-Bed Fusion (LPBF), Additive Manufacturing, Modelling, Temperature Gradient, Residual Stress and Distortion

\begin{abstract}
The build-up of residual stresses in a part during laser powder-bed fusion (LPBF) provides a significant limitation to the adoption of this process. These residual stresses may cause a part to fail during a build or fall outside the specified tolerances after fabrication. Defectfree production of metallic parts using LPBF requires process optimization, as a crucial step, for effective usage of the process. Development of a numerical model to accurately predict the induced residual stresses and distortion during the LPBF process is of great interest as it allows to effectively investigate the influence of processing parameters on the quality of the parts. In this work, we developed a novel high-fidelity finite element (FE) model based on the inherent strain (local-global) approach to simulate the build process and calculate the residual stress and distortion for Hastelloy X specimens built with a continuous scan strategy. Conclusions from the thermomechanical simulations showed good agreement with X-ray diffraction measurements and 3D scanning data used to determine the residual stresses and distortions in the parts.
\end{abstract}

\section{INTRODUCTION}

Additive manufacturing (AM) is a layer-by-layer fabrication technology poised to bring about a revolution in the way products are designed, manufactured, and integrated. The greatest impacts will come through the AM of metal parts. This technology has gained significant industry interest due to its ability to create parts consolidation and complex geometries with customizable material properties. Among metal AM processes, laser powder bed fusion (LPBF) is the major technology as it represents around $80 \%$ of metal AM equipment installed globally [1]. Although being formerly used to produce prototypes, LPBF is more and more foreseen to manufacture near-net-shape structural components with complex geometries. Nevertheless, wide industrial applications of LPBF are hindered by several limitations, including porosity defects resulted from lack of fusion, keyhole collapse, balling, and residual stress which causes distortion and failure of the final products due to high thermal gradients [2-4]. Therefore,

(C) 2021 National Research Council of Canada 
machine process parameter optimization becomes a critical task. Understanding and mastering the phenomena that occur during the printing process remains key to reduce trial-and- errors, and, in the end, maximize build efficiency. This is the main challenge facing LPBF manufacturers when trying to develop new parts or use new materials as the process behavior is highly dependent on the process parameters, geometry and material [5]. Physical phenomena associated with LPBF processes involve multiple highly coupled physics, including phase change (melting/solidification and vaporization) [6], heat and mass transfer, thermo-mechanical interactions occurring at various time and length scales [7]. From a scientific standpoint, physics-based modelling and simulation of the LPBF remain a formidable challenge and minimizing defects occurrence in metallic parts requires an understanding of the underlying AM-processing physics that are inherent in printing components.

Unfortunately, no single model is capable of simulating all aspects of LPBF due to the large range of length and time scales inherent to the problem. Powder-scale models have shown the ability to predict porosity by modeling the complex physical phenomena associated with laser melting and consolidation of a single scan track or single layer [7-9]. These models resolve time scales on the order of $n s$ to $m s$ and length scales from $\mu m$ to $\mathrm{mm}$ and are not currently practical for simulation of an entire part via LPBF. Macroscale models for predictions of residual stress and distortion use many assumptions to speed up the calculations. The two main classes of these part-scale models are inherent strain-based codes and thermomechanical codes. Inherent strain-based codes employ a specified residual strain field that is applied layer by layer when simulating an entire part. The simulation solves the solid mechanics problem only without considering the balande of thermal energy.
In this work, a particular effort has been made to develop an accurate model for the heat
input as it has a significant influence on temperature distribution and has a direct correlation
with the melt pool shape. A 3D non-linear transient thermo-viscoplastic FE model is then
developed to predict the plastic strains in a multi-tracks pattern on powder substrate. The inherent strain obtained from thermomechanical simulation is subsequently applied to an entire

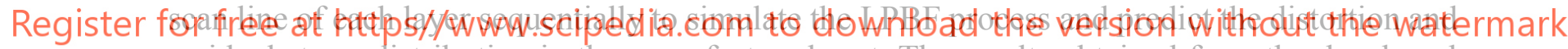
residual stress distribution in the manufactured part. The results obtained from the developed multi-scale FE model are compared with those obtained experimentally and with previously published FE simulations.

\section{MATHEMATICAL FORMULATIONS}

In this section, a brief discussion on underlying governing equations addressing the heat transfer and structural analysis is provided. Simulation of the LPBF process is a complex multiphysics problem involving highly nonlinear dynamically coupled thermomechanical phenomenon which demands an enormous computational effort. For the sake of completeness, a viscoplastic model that considers the thermal history of the component during the LPBF process is developed. The model assumes that at high temperatures, the material exhibits creep and time-dependent viscoplastic deformation behavior simultaneously.

\subsection{Heat Transfer Governing Equation}

The governing expression for 3D heat transfer process can generally be written as follows: 


$$
\rho(\mathrm{T}) \mathrm{c}_{\mathrm{p}}(\mathrm{T}) \frac{\partial \mathrm{T}}{\partial \mathrm{t}}=\nabla \cdot(\mathrm{k}(\mathrm{T}) \nabla \mathrm{T})+\overline{\mathrm{Q}}
$$

where $\rho$ is the density of the material, $c_{p}$ is the specific heat, $k$ is the thermal conductivity, $T$ is the temperature and $\bar{Q}$ is the heat transferred to the material by the laser beam. The density of the material, specific heat and thermal conductivity are temperature-dependent and make thermal equations nonlinear.

During the powder-bed fusion process, the heat supplied by the laser beam is conducted to the edges of the workpiece. Therefore, heat conduction is an important parameter to consider in the thermal analysis. Additionally, heat losses through convection and radiation should be taken into account.

Convective heat losses, $\mathrm{q}_{\mathrm{c}}$, were considered as follows:

$$
\mathrm{q}_{\mathrm{c}}=-\mathrm{h}_{\mathrm{c}}\left(\mathrm{T}_{\mathrm{s}}-\mathrm{T}_{0}\right)
$$

where $h_{c}$ is the convective heat transfer coefficient, $T_{S}$ is the surface temperature, and $T_{0}$ is the ambient temperature.

Radiative heat losses, qR, were accounted for by using Stefan- Boltzmann law:

$$
\mathrm{q}_{\mathrm{R}}=\sigma \varepsilon\left(\mathrm{T}_{\mathrm{s}}^{4}-\mathrm{T}_{0}^{4}\right)
$$
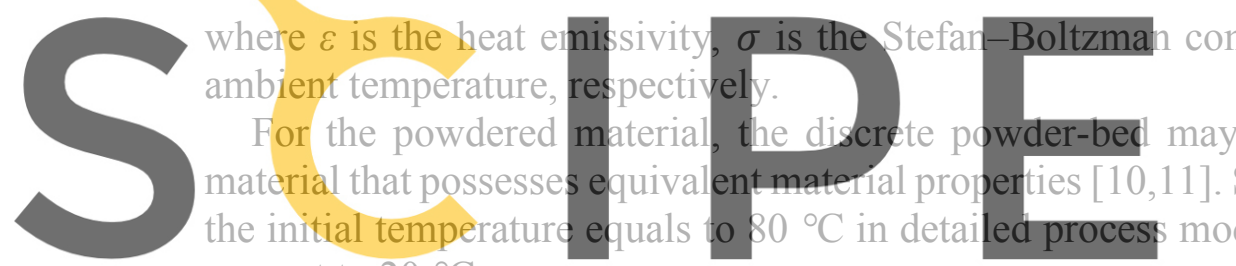
was set to $20^{\circ} \mathrm{C}$.

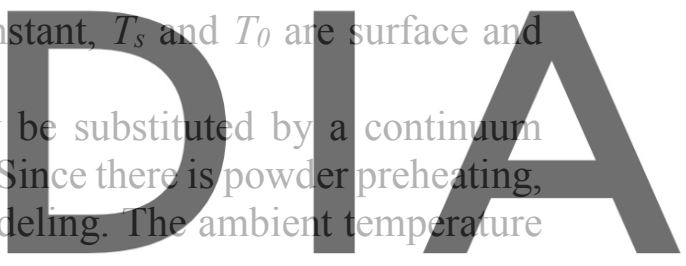

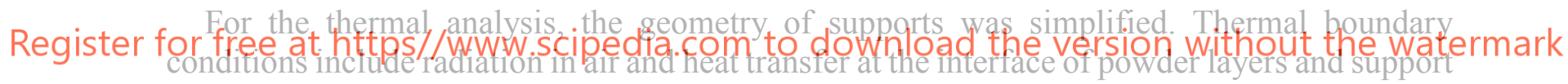
material. Radiation heat loss to the ambient environment was applied to all exterior surfaces.

\subsection{Heat source model}

To conduct an accurate numerical simulation, the parameters of the heat source model which have a significant influence on temperature distribution and melt pool shape should be initially determined. In this work, a particular effort has been made to develop an accurate model for the heat input since it has a significant influence on temperature distribution and has a direct correlation with the melt pool shape. A 3D printing EOS M290 machine was employed for the fabrication of Hastelloy X test coupons. Single path scans were conducted to form single beads on a $40 \mu \mathrm{m}$ layer thickness of powder layer. For the process parameter of $200 \mathrm{~W}$ power and $1000 \mathrm{~m} / \mathrm{s}$ speed, 9 scans and therefore, 9 single beads were produced. All the single beads were sectioned, polished, and etched for measuring geometrical features of melt pools.

A Gaussian heat source by a volume density of energy shown in Figure 1 is applied which moves along the scanning trajectory. 


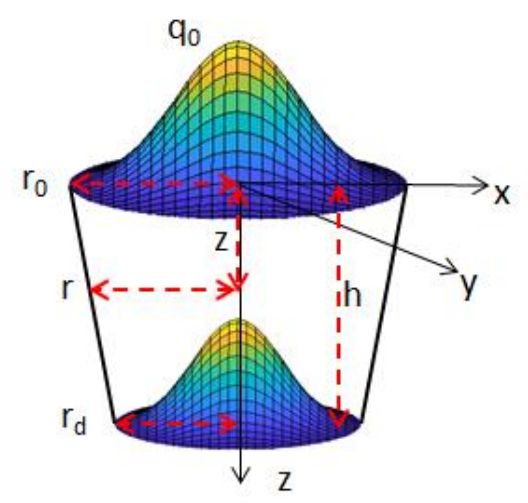

Figure 1: Gaussian heat source model.

The conical volumetric heat source is represented as Eq. (4) in which the parameters include:

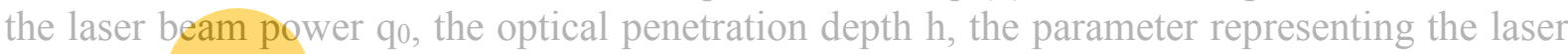
spot size $r_{z}$, and the position $(x, y, z)$ with respect to the heat source center location:

$$
\mathrm{q}_{\mathrm{vol}}(\mathrm{x}, \mathrm{y}, \mathrm{z})=\mathrm{q}_{0} \exp \left[-\frac{2\left(\mathrm{x}^{2}+\mathrm{y}^{2}\right)}{\mathrm{r}_{\mathrm{z}}^{2}}\right]
$$
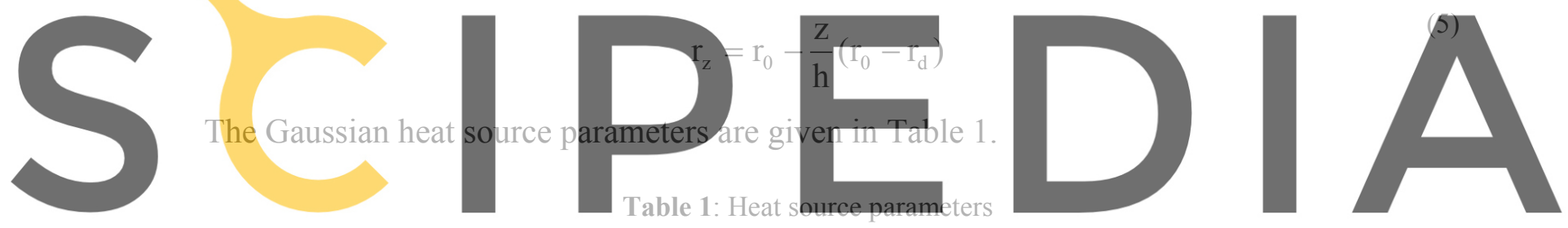

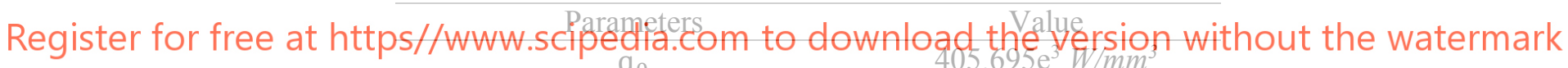

\begin{tabular}{cc}
$\mathrm{r}_{0}$ & $50 \mu \mathrm{m}$ \\
$\mathrm{r}_{\mathrm{d}}$ & $40 \mu \mathrm{m}$ \\
\hline $\mathrm{h}$ & $50 \mu \mathrm{m}$ \\
\hline Absorption coefficient [12] & 0.3 \\
\hline
\end{tabular}

Several iterations with different values for laser absorption coefficient and anisotropic thermal conductivity of powder were made to ensure the alignment between micro cross-section melt zone and melt pool determined by numerical simulations. Figure 2 shows the maximum temperature and the fusion zones from the micro cross-section obtained from optical microscopy and the numerical results. 


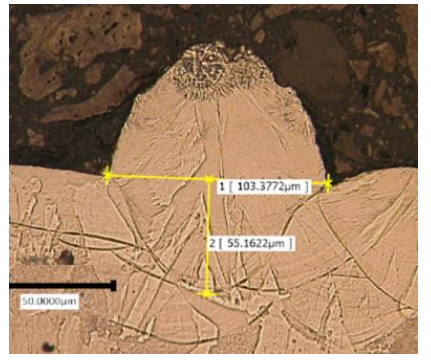

(a)

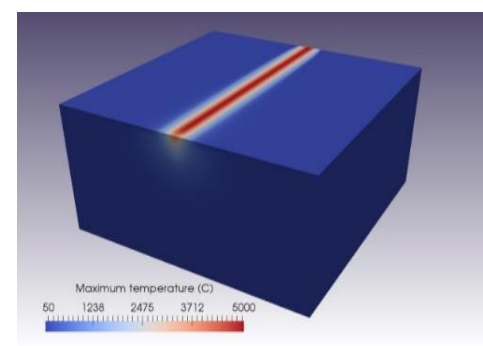

(b)

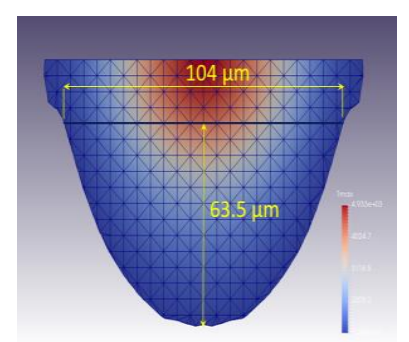

(c)

Figure 2: (a) measured fusion zone; (b) temperature distribution; (c) simulated fusion zone.

\subsection{Elasto-viscoplastic model for stress and deformation analysis}

To explain the time-dependent stress-strain behavior subjected to a high strain rate occurring in LPBF additive manufacturing, an elasto-viscoplastic model as developed by Perzyna $[13,14]$ is used. For nonlinear continuum problems, it is assumed that there is no irreversible flow if the yield criterion is not satisfied. The total strain, $\varepsilon$, can be separated into elastic, $\varepsilon_{\mathrm{el}}$, thermal, $\varepsilon_{\mathrm{th}}$

, phase change, $\varepsilon_{\mathrm{tr}}$, and viscoplastic, $\varepsilon_{\mathrm{vp}}$, components, so that the total strain rate can be expressed as [15-17]:
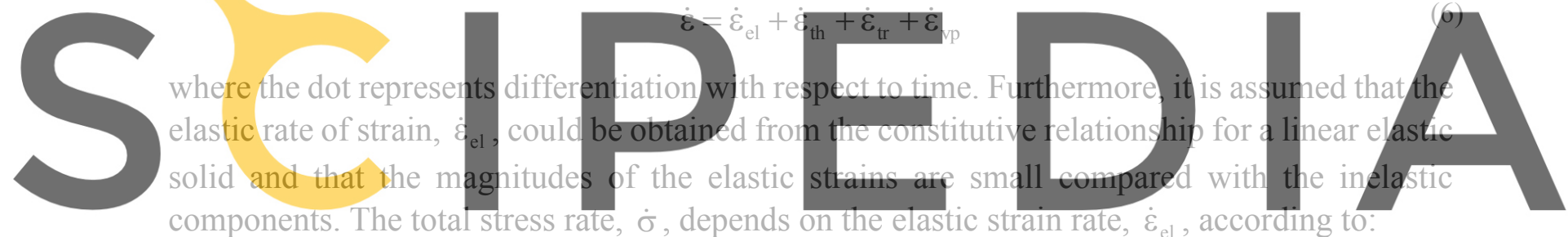

components. The total stress rate, $\dot{\sigma}$, depends on the elastic strain rate, $\dot{\varepsilon}_{\mathrm{el}}$, according to

Register for free at https//www.scipedia.com to-dewnload the version without the watermark

where D is the elasticity matrix.

The viscoplastic behavior is governed by a scalar yield condition of the form

$$
\mathrm{F}\left(\sigma, \varepsilon_{\mathrm{vp}}\right)=\mathrm{f}\left(\sigma, \varepsilon_{\mathrm{vp}}\right)-\sigma_{\mathrm{y}}=0
$$

where, $\sigma_{\mathrm{y}}$, is the uniaxial yield stress which can be determined experimentally from uniaxial tensile tests. It is assumed that viscoplastic flow occurs for values of $f>\sigma_{y}$.

Hereafter in this analysis, we restrict ourselves to a consideration of Von Mises yield criterion in which:

$$
\mathrm{f}=\sigma_{\mathrm{eq}}=\sqrt{\frac{3}{2} \mathrm{~s}_{\mathrm{ij}} \mathrm{s}_{\mathrm{ij}}}
$$

In Eq. (9), $\sigma_{\mathrm{eq}}$, is the equivalent stress and $\mathrm{s}_{\mathrm{ij}}$ are components of the deviatoric stresses and are given by: 


$$
\mathrm{s}_{\mathrm{ij}}=\sigma_{\mathrm{ij}}-\frac{1}{3} \sigma_{\mathrm{mm}} \delta_{\mathrm{ij}}
$$

For relatively rapid processes, the uniaxial yield stress, $\sigma_{y}$, in Eq. (8) may be given by the Johnson-Cook viscoplastic model [18], expressed as:

$$
\sigma_{\mathrm{y}}\left(\mathrm{h}, \bar{\varepsilon}_{\mathrm{vp}}, \dot{\bar{\varepsilon}}_{\mathrm{vp}}, \mathrm{T}\right)=\left(\sigma_{\mathrm{y}}^{0}+\mathrm{h} \bar{\varepsilon}_{\mathrm{vp}}^{\mathrm{N}}\right)\left(1+\mathrm{C} \ln \left(\frac{\dot{\bar{\varepsilon}}_{\mathrm{vp}}}{\dot{\bar{\varepsilon}}_{0}}\right)\right)\left(1-\left(\frac{\mathrm{T}-\mathrm{T}_{0}}{\mathrm{~T}_{\mathrm{m}}-\mathrm{T}_{0}}\right)^{\mathrm{M}}\right)
$$

where, $\sigma_{\mathrm{y}}^{0}$, is the initial uniaxial yield stress, $\mathrm{h}$ is the hardening coefficient, $\mathrm{N}$ is the hardening exponent, $T_{m}$ is the melting temperature, $T_{0}$ is the reference temperature, $M$ is the thermal softening exponent material parameter, $\dot{\bar{\varepsilon}}_{\mathrm{vp}}$ is the equivalent viscoplastic strain rate, $\dot{\bar{\varepsilon}}_{0}$ is the equivalent viscoplastic strain rate threshold and $\bar{\varepsilon}_{\mathrm{vp}}$ is the effective or equivalent viscoplastic strain, defined as:

$$
\bar{\varepsilon}_{\mathrm{vp}}=\sqrt{\frac{2}{3}}\left\{\left(\varepsilon_{\mathrm{ij}}\right)_{\mathrm{vp}}\left(\varepsilon_{\mathrm{ij}}\right)_{\mathrm{vp}}\right\}^{\frac{1}{2}}
$$

In the Johnson-Cook model Eq. (11), the first part is used to describe the work-hardening effect, the second one for the strain-rate effect and the last one for the temperature effect.

\section{The strain hardening} equivalent stress, $\sigma_{\text {eq }}$
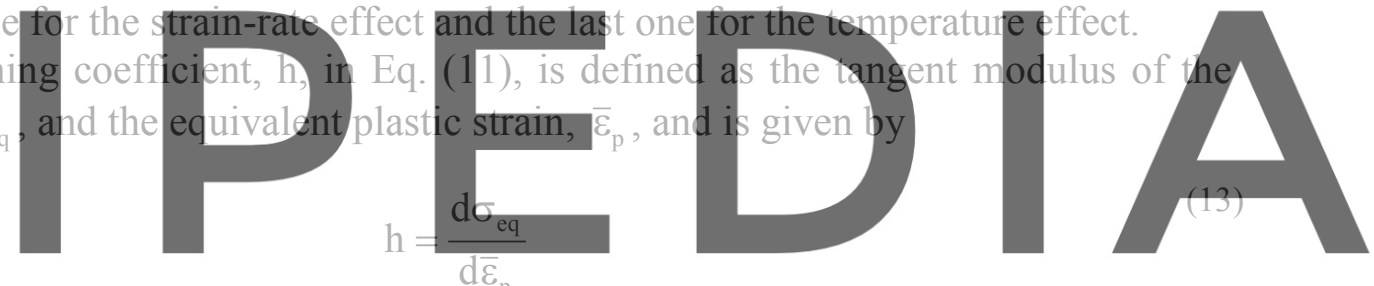

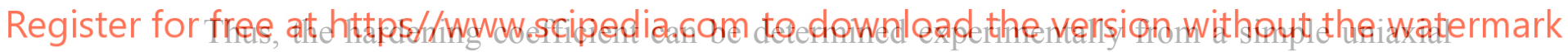
yield test. It is now necessary to choose a specific law defining the viscoplastic strains. The simplest option is one in which the viscoplastic strain rate depends on the current stresses, so that $[13,14]$

$$
\dot{\varepsilon}_{\mathrm{vp}}=\eta\langle\Phi(\mathrm{F})\rangle \frac{\partial \mathrm{F}}{\partial \sigma}
$$

where, $\eta$, is a fluidity parameter controlling the plastic flow rate, $\frac{\partial F}{\partial \sigma}$ is the flow vector, $\Phi(F)$ is a positive monotonic increasing function for $\mathrm{F}>0$ and the notation $\langle\ldots\rangle$ implies

$$
\begin{aligned}
& \langle\Phi(F)\rangle=\Phi(F) \text { for } F>0 \\
& \langle\Phi(F)\rangle=0 \quad \text { for } F \leq 0
\end{aligned}
$$

The above theory of viscoplasticity is essentially based on the concept that the stress beyond the pseudo-static yield surface, $\mathrm{F}=0$, called excess stress, provides the viscosity: 


$$
\Phi(\mathrm{F})=\mathrm{F}
$$

In this work, the yield function of the form given by Eq. (8) is employed with the viscoplastic constitutive equation, Eq. (14), instead of the Perzyna's original form:

$$
\mathrm{F}\left(\sigma, \varepsilon_{\mathrm{vp}}\right)=\frac{\mathrm{f}\left(\sigma, \varepsilon_{\mathrm{vp}}\right)-\sigma_{\mathrm{y}}}{\sigma_{\mathrm{y}}}=0
$$

In fact, when the yield stress of the material tends to zero $\left(\sigma_{y} \rightarrow 0\right)$ at high temperature, especially for liquid above the melting point, an infinitely large inelastic strain rate is achieved due to a very small amount of excess stress. Thus, the reduced constitutive equation, Eq. (14), with the yield function Eq. (8) may describe the material behavior over a wide range of temperature [19].

Thermal and phase change rates of strain are given respectively by:

$$
\dot{\varepsilon}_{\text {th }}=\alpha \dot{T}
$$

and

$$
\dot{\varepsilon}_{\mathrm{tr}}=\beta \dot{\mathrm{f}}_{\mathrm{s}}
$$

where, $\alpha$, is the linear thermal expansion coefficient, $\mathrm{T}$ is the temperature field, $\beta$ is the linear

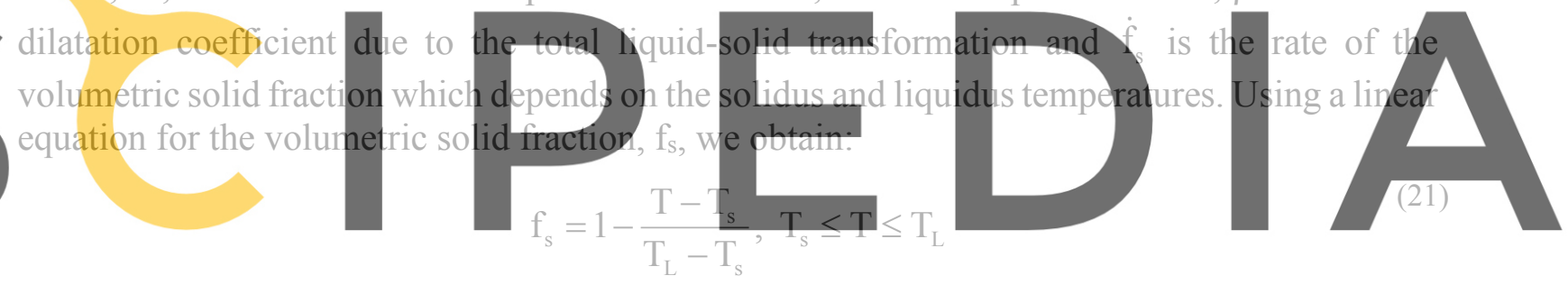

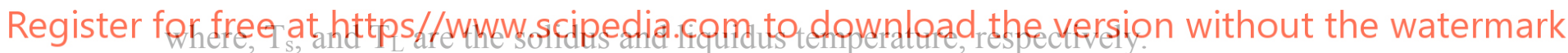

\subsection{Time integration and FEM implementation}

With the strain rate law expressed by Eq. (14), we can define a strain increment, $\Delta \varepsilon_{\mathrm{vp}}^{\mathrm{n}}$, occurring in a time interval, $\Delta \mathrm{t}_{\mathrm{n}}=\mathrm{t}_{\mathrm{n}+1}-\mathrm{t}_{\mathrm{n}}$, using an implicit time-stepping scheme as:

$$
\Delta \varepsilon_{\mathrm{vp}}^{\mathrm{n}}=\left[(1-\theta) \dot{\varepsilon}_{\mathrm{vp}}^{\mathrm{n}}+\theta \dot{\varepsilon}_{\mathrm{vp}}^{\mathrm{n}+1}\right] \Delta \mathrm{t}_{\mathrm{n}}
$$

For $\theta=0$, we obtain the fully explicit Euler time integration while, $\theta=1$, gives a fully implicit Euler time integration scheme. Limited Taylor series expansion of the viscoplastic strain rate leads to:

$$
\dot{\varepsilon}_{\mathrm{vp}}^{\mathrm{n}+1}=\dot{\varepsilon}_{\mathrm{vp}}^{\mathrm{n}}+\frac{\partial \dot{\varepsilon}_{\mathrm{vp}}^{\mathrm{n}}}{\partial \sigma^{\mathrm{n}}} \Delta \sigma^{\mathrm{n}}=\dot{\varepsilon}_{\mathrm{vp}}^{\mathrm{n}}+\mathrm{H}^{\mathrm{n}} \Delta \sigma^{\mathrm{n}}
$$

Combining Eq. (22) and Eq. (23), we may express the viscoplastic strain increment as: 


$$
\Delta \varepsilon_{\mathrm{vp}}^{\mathrm{n}}=\Delta \mathrm{t}_{\mathrm{n}} \dot{\varepsilon}_{\mathrm{vp}}^{\mathrm{n}}+\theta \Delta \mathrm{t}_{\mathrm{n}} \mathrm{H}^{\mathrm{n}} \Delta \sigma^{\mathrm{n}}
$$

where

$$
\dot{\varepsilon}_{\mathrm{vp}}^{\mathrm{n}}=\frac{3}{2} \eta \mathrm{F}^{\mathrm{n}} \frac{\mathrm{s}^{\mathrm{n}}}{\sigma_{\mathrm{eq}}^{\mathrm{n}}}
$$

The viscoplastic strain at time $t_{n+1}$ is:

$$
\varepsilon_{\mathrm{vp}}^{\mathrm{n}+1}=\varepsilon_{\mathrm{vp}}^{\mathrm{n}}+\Delta \varepsilon_{\mathrm{vp}}^{\mathrm{n}}
$$

Using the incremental form of Eq. (7), we obtain the stress change, $\Delta \sigma^{\mathrm{n}}$, occurring in the time interval, $\Delta \mathrm{t}_{\mathrm{n}}$, as

$$
\Delta \sigma^{\mathrm{n}}=\mathrm{D} \Delta \varepsilon_{\mathrm{e}}^{\mathrm{n}}=\mathrm{D}\left(\Delta \varepsilon^{\mathrm{n}}-\Delta \varepsilon_{\text {th }}^{\mathrm{n}}-\Delta \varepsilon_{\mathrm{tr}}^{\mathrm{n}}-\Delta \varepsilon_{\mathrm{vp}}^{\mathrm{n}}\right)
$$

The total strain increment in terms of displacement increment is written as:

$$
\Delta \varepsilon^{\mathrm{n}}=\mathrm{B} \Delta \mathrm{d}^{\mathrm{n}}
$$

where $\mathrm{B}$ is strain matrix and $\Delta \mathrm{d}^{\mathrm{n}}$ is the displacement increment. Therefore, the stress increment given by Eq. (27) can be expressed as:
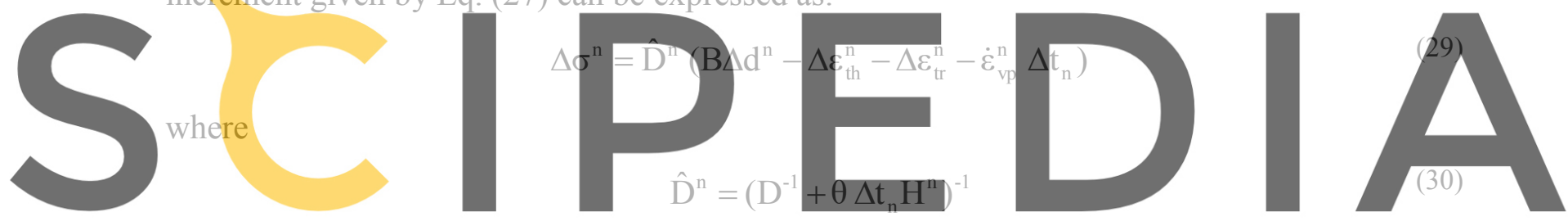

At each time step, the steady-state condition or the convergence is monitored by checking Register for free at https/Lwww.scipedia.com to download the version without the wa.termark

\section{less than a given tolerance.}

Theoretical restrictions on the time step length have been provided by Cormeau [20] for specific forms of viscoplastic flow rule and for explicit time integration. The stability criterion for a Von Mises material in the quasi-linear situation, $\Phi(F)=F$, is given by:

$$
\eta \Delta \mathrm{t}_{\mathrm{n}} \leq \frac{4(1+v)}{3 \mathrm{E}}
$$

where E and $v$ are Young's modulus and Poisson's ratio, respectively.

The incremental form of the equations of equilibrium to be satisfied at any instant of time, $t_{n}$, are:

$$
\int_{\Omega}\left[\mathrm{B}^{\mathrm{n}}\right]^{\mathrm{T}} \Delta \sigma^{\mathrm{n}} \mathrm{d} \Omega+\Delta \mathrm{r}^{\mathrm{n}}=0
$$

where, $\Delta \mathrm{r}^{\mathrm{n}}$, is the vector of equivalent nodal loads increment due to applied surface tractions and body forces. In Eq. (32) the notation, $\mathrm{B}^{\mathrm{n}}$, is employed to denote the possibility that the 
strain matrix may not be constant throughout the solution. As the simulation of the LPBF process involves the coupled solution, at each time step, of thermal and elasto-viscoplastic equations, the calculation time quickly becomes prohibitive as soon as the computational domain becomes large. Thus, we appeal to the local-global method that we describe briefly below.

\subsection{Inherent strain (local-global) method}

The inherent strain (local-global) method developed by Souloumiac [21] needs plastic strains calculated from a local 3D model with a thermo-viscoplastic method as input. The simulation is performed on a small computational domain (local method), that represents the thermal and mechanical conditions of the rest of the structure. Therefore, the local analysis includes all the physics involved in the process. The plastic strains, $\varepsilon_{\mathrm{p}}$, calculated from the local model are employed in a layer-by-layer as initial strains in the global 3D model as shown in Figure 3.
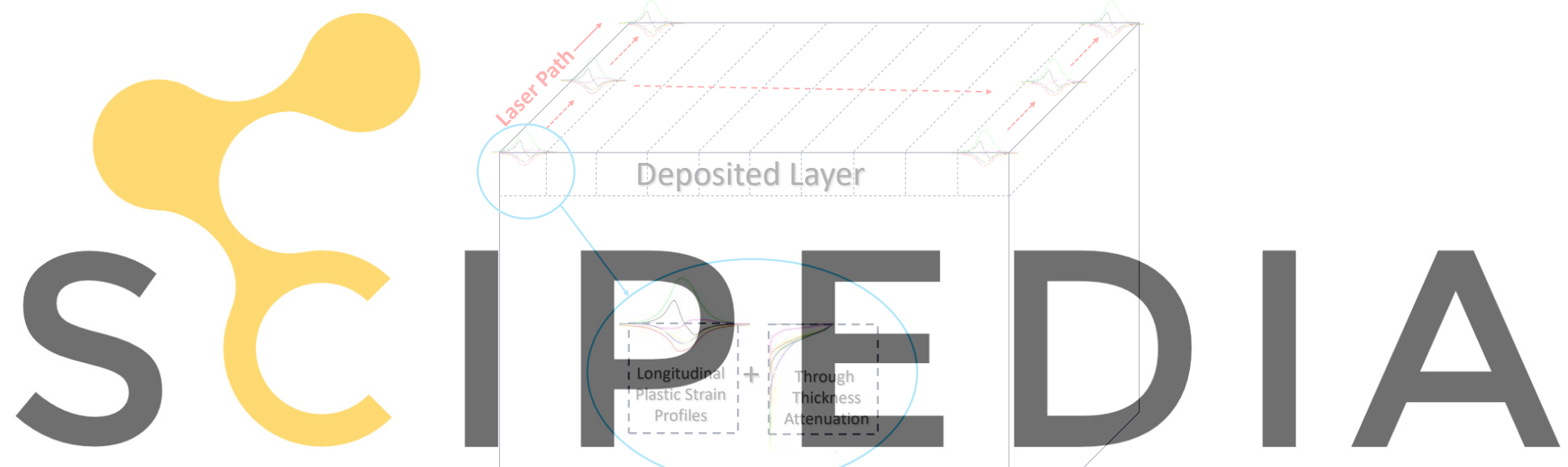

Register for free at https//www.scipedia.eophttfolownload the version without the watermark

Figure 3: Initial plastic strain are imposed by incrementally applying local strain over all laser tracks and layers.

The stress of the complete structure is obtained by elastic computation, as:

$$
\Delta \sigma^{\mathrm{n}}=\mathrm{D} \Delta \varepsilon_{\mathrm{e}}^{\mathrm{n}}=\mathrm{D}\left(\Delta \varepsilon^{\mathrm{n}}-\Delta \varepsilon_{\mathrm{p}}^{\mathrm{n}}\right)
$$

The global model can be discretized in a relatively coarse fashion. In the calculation of the global model, it is possible to indicate the laser paths and study the influence of the laser path on the residual stresses and the distortions.

\section{CASE STUDIES}

\subsection{Single cantilever beam: comparison of experimental and numerical results}

A comprehensive simulation work on the distortion of the cantilever beam after cutting the support has been studied in [22]. The schematic of the combined part, support and substrate is 
shown in Figure 4, along with the finite element mesh used for the global model analysis.
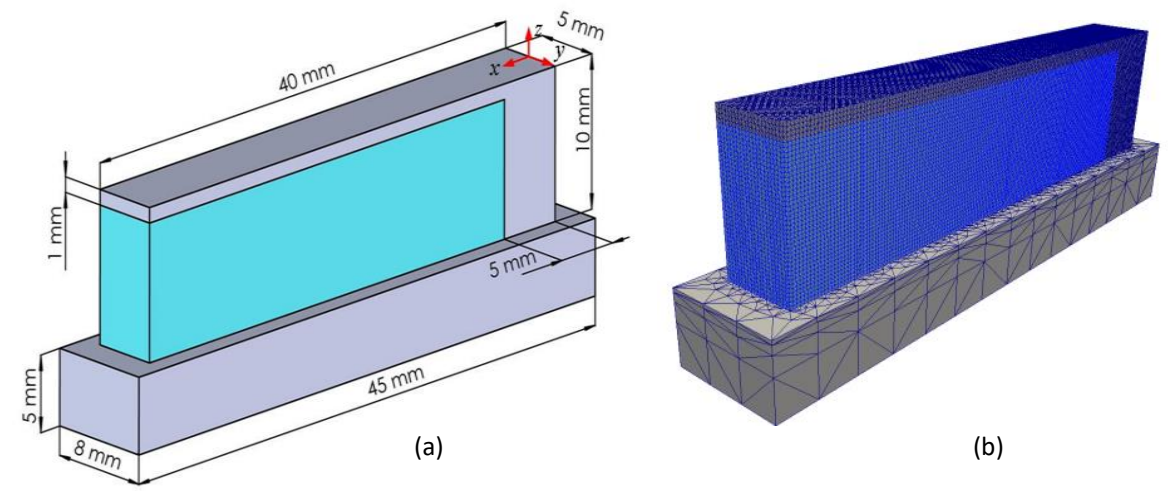

Figure 4: Single cantilever beam: (a) schematic of the part, support and substrate from Ref [22] and (b) FEM model.

\section{The cantilever is made from Hastelloy X with Young's modulus of $190 \mathrm{GPa}$ and Poisson's} ratio of 0.3 [22]. Following the geometry description given in [22] the top of the support domain is connected with the cantilever, while the right side of the support domain has a one-element gap from the cantilever. The local-global model has been employed and the distortion of the

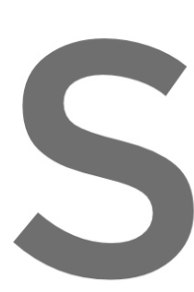
cantilever beam is det
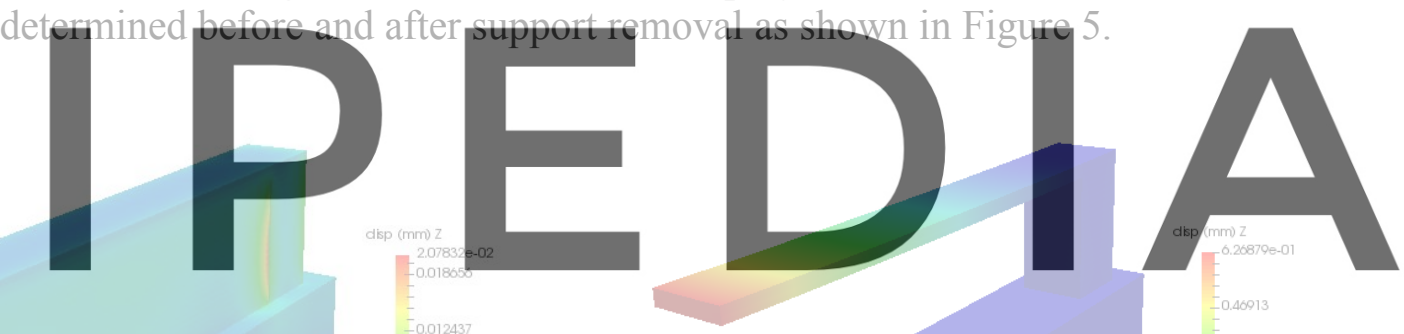

Register for free at https//www.scipedia.com to download the version without the watermark
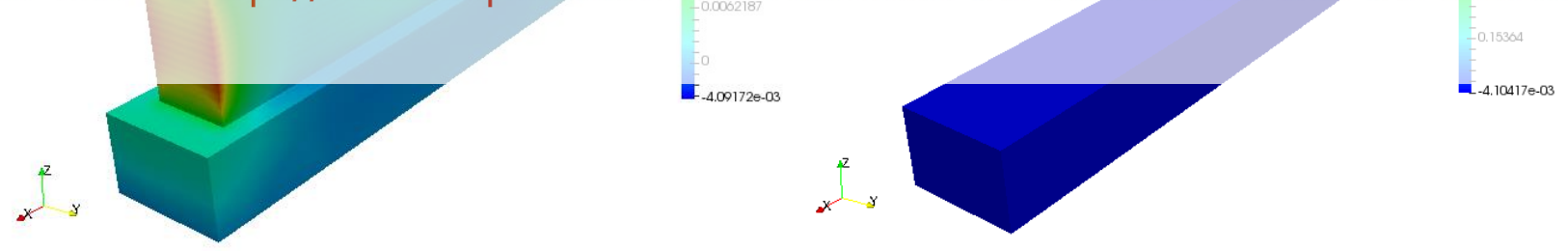

Figure 5: Deflection of the cantilever beam: (a) before cutting of the support and (b) after cutting of the support.

The predicted distortion using thermo-viscoplastic, inherent strain (local-global) method, before and after removing of the substrate, is shown in Figure 6. 


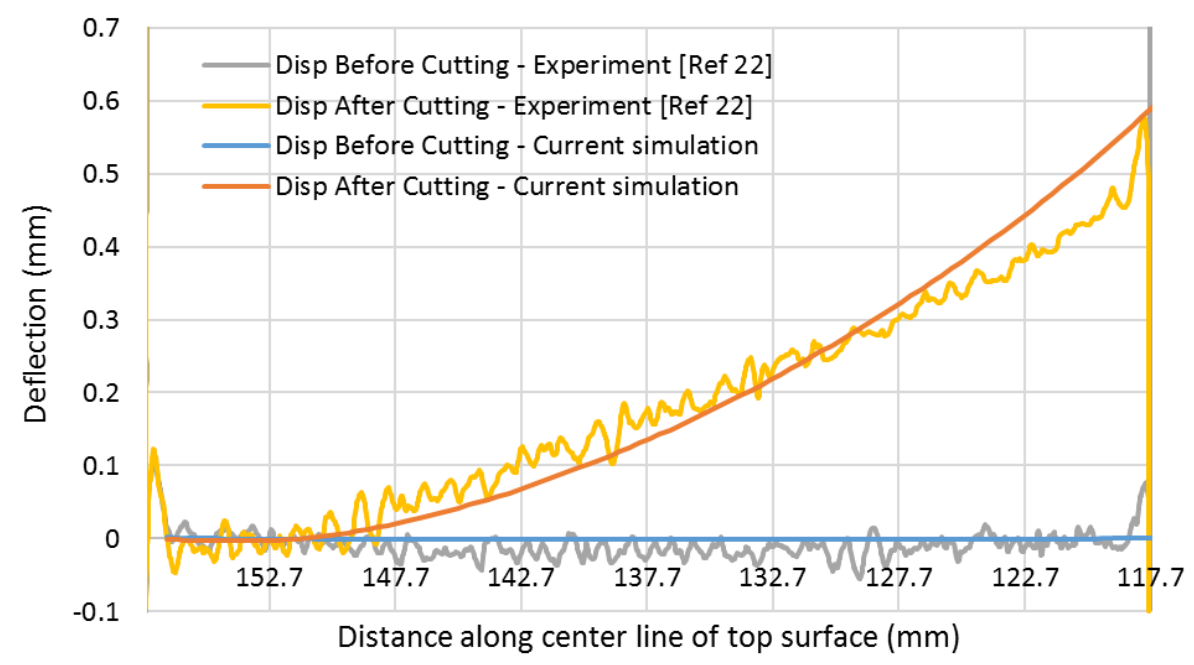

Figure 6: Displacement deflection along the center line at the top surface of the cantilever beam after cutting of the support. Comparison between experiment measurements [22] and current numerical simulation results.

The inherent strain method correctly predicts the displacement distortion with an error of about $0.6 \%$ at a distance of $117.7 \mathrm{~mm}$ along the center line of the top surface when comparing with the experimental measurement as shown in Figure 6.

\subsection{Double cantilever beam}

The results obtained by the proposed FEM were also compared with modeling results on double cantilever produced by LPBF reported by Chen et al. [23]. The material data are those of Inconel 718 [23]. Schematic of the part and the FEM model are shown in Figure 7.

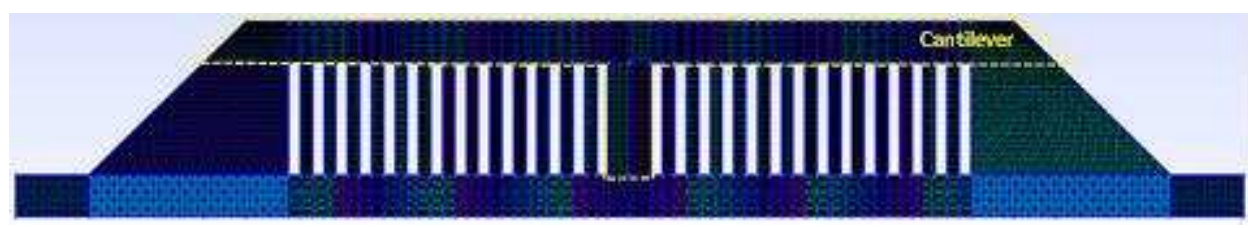

Figure 7: FEM model of the double cantilever beam: schematic of the part, support and substrate.

The distortion profile computed from the proposed inherent strain method (local-global) is determined and compared with that obtained by Chen et al [23]. In both simulations, large distortion is observed along the connections between the beam and support columns.

The distortion profile and magnitude before cutting off the supports as obtained from the proposed inherent strain method is shown in Figure 8. 


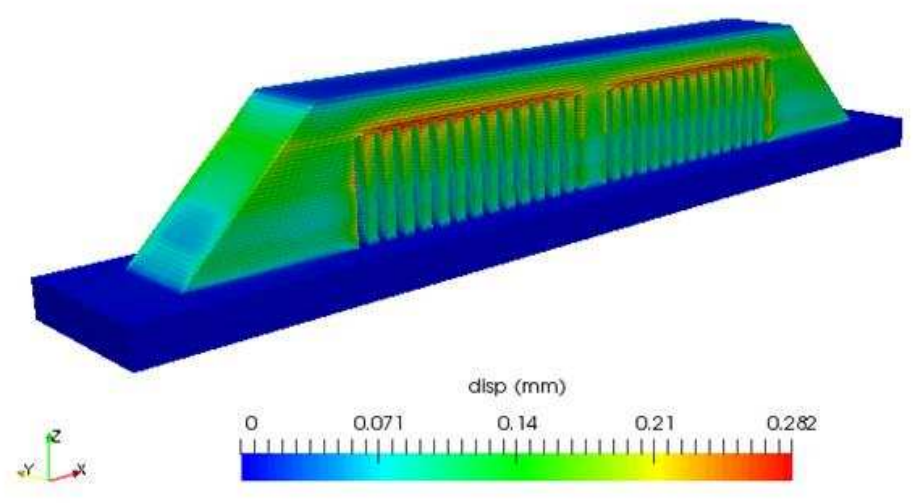

Figure 8: Deflection of the cantilever beam before cutting of the support.

After cutting the supports, the strains are released, and the cantilever is distorted by the spring back effect (see Figure 9).
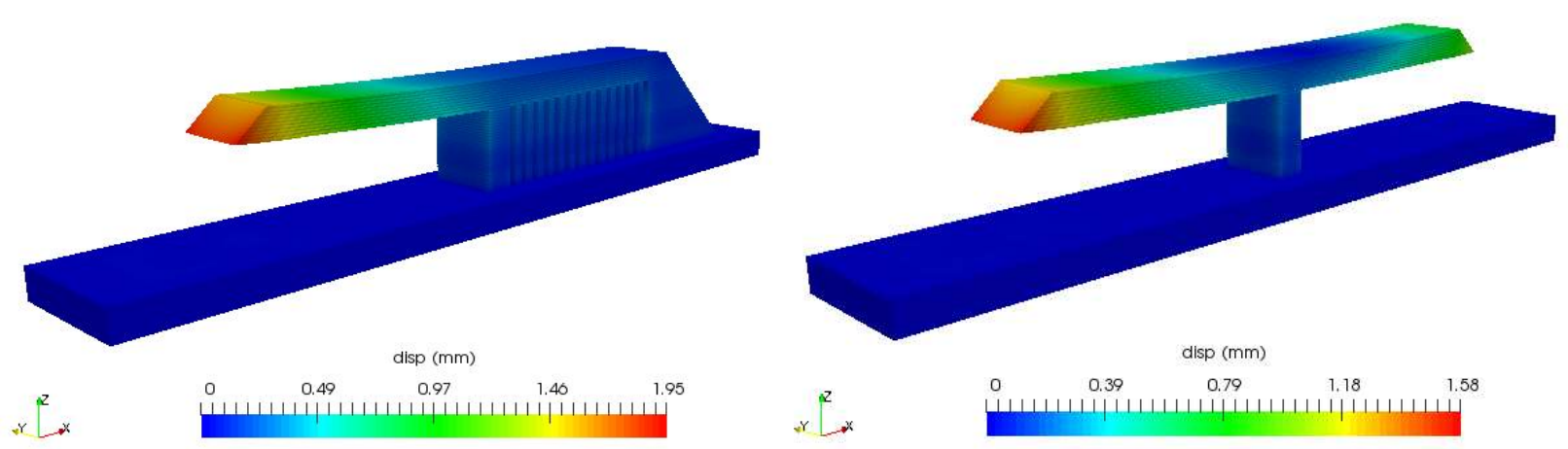

Figure 9: Cantilever beam distortion: (a) after cutting of the left support and (b) after cutting of the right support.

The elastic strain predictions before and after support removal are almost identical to those reported by Chen et al. [23].

Residual stresses have been studied before and after removing the support structure as illustrated in Figures 10 and 11. The obtained results show a very good agreement with those of Chen et al. [23]. 


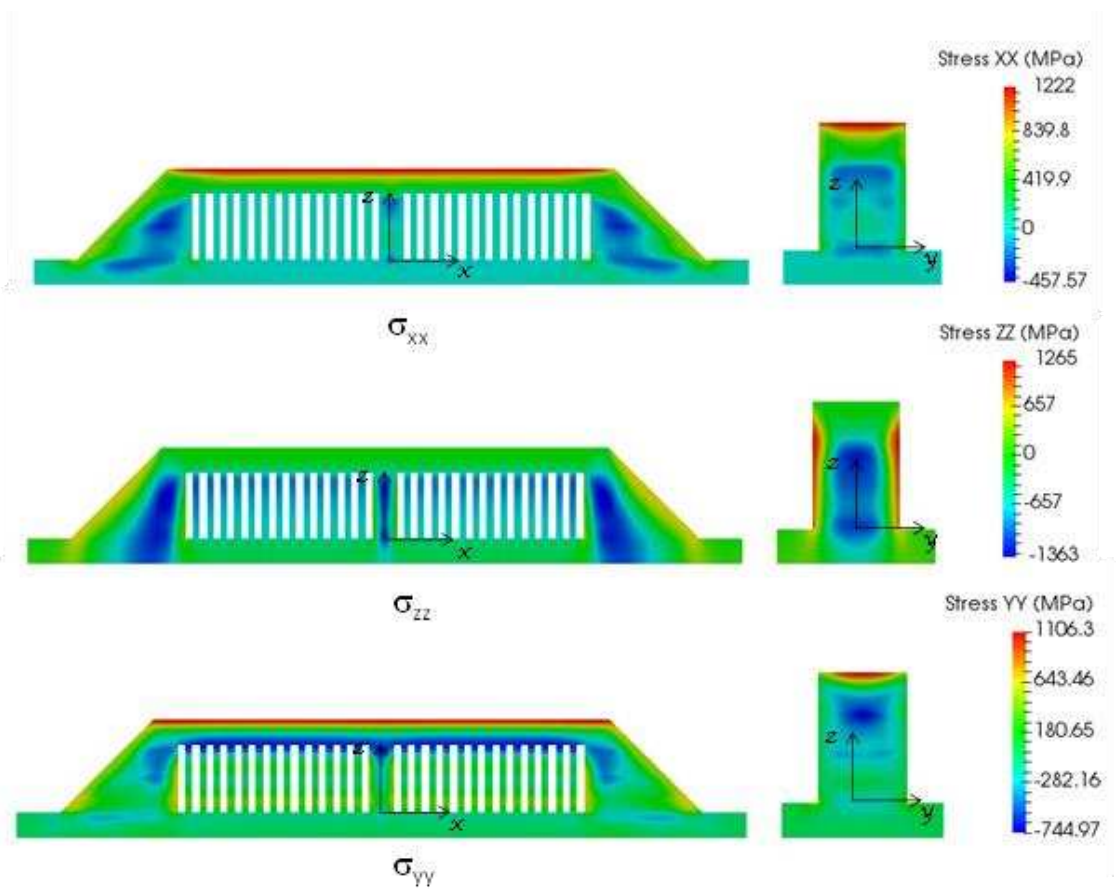

Figure 10: Residual stresses before cutting.

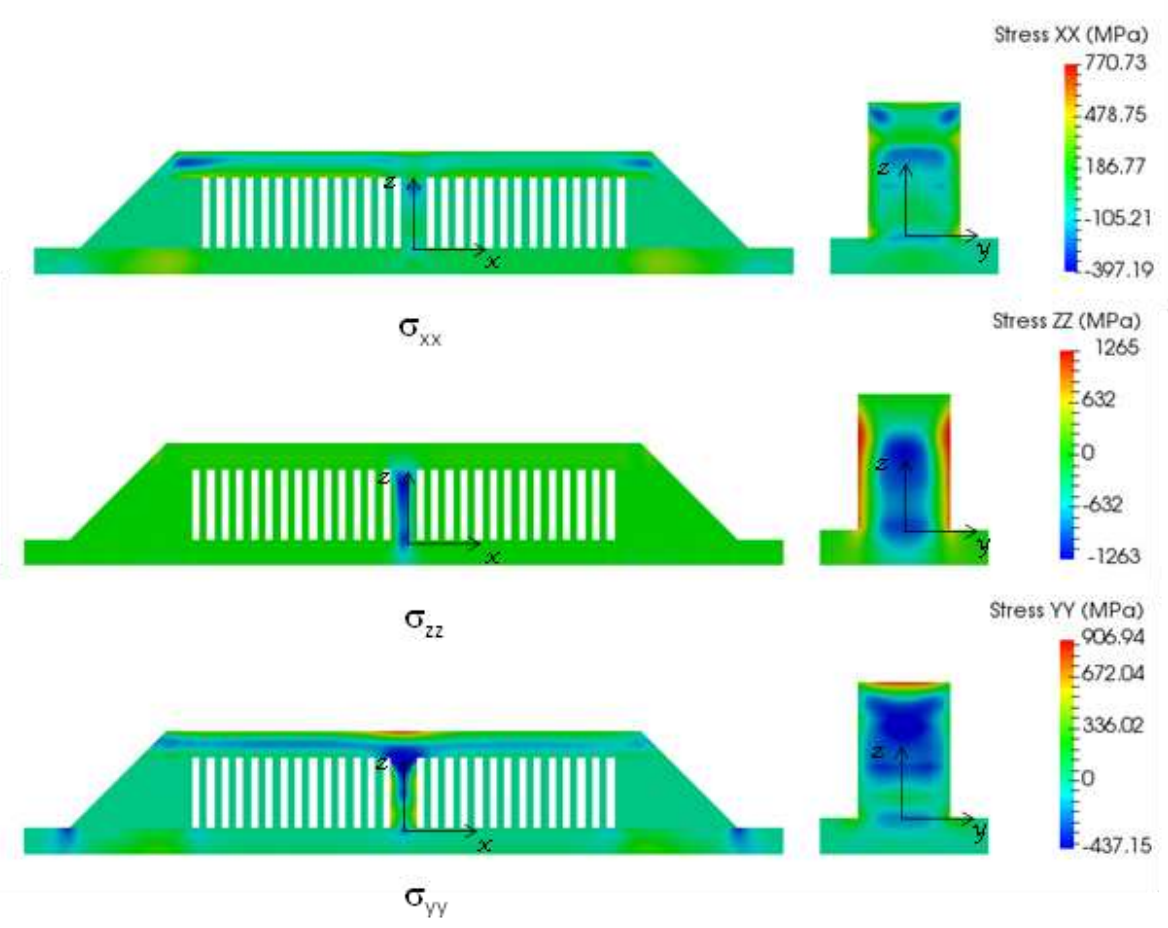

Figure 11: Residual stresses after cutting.

The maximum tensile residual stress was found on the surface of the part which is caused by 
the contraction of the molten material after cooling. The tensile stress decreases as the depth increases. The predicted residual stress profile was very similar to the typical simplified residual stress profile in-depth direction [24-25].

\section{CONCLUSIONS}

A novel multiscale three-dimension numerical model has been developed to accurately predict the induced residual stresses and distortion during the LPBF process. On the basis of cross-sections obtained from the SEM process, a Gaussian heat source distribution with calibrated parameters has been adopted for the coupled thermo-mechanical simulation. The plastic strain profile developed by the microscale model (local) has then been utilized as an input for the macroscale model (local-global). The novel inherent strain (local-global) approach has been employed to predict the residual stress and distortion of part scale single and double cantilever beams. The inherent strain method well predicted the residual stress and distortion of the printed cantilevers. Tensile residual stresses are found on the top surface of the part. Reduction of residual stress in-depth direction is observed. The residual stress profile simulated by the proposed method on the single and double cantilever beams correlates well with other experimental and numerical studies found in the existing literature. The predicted distortions are comparable to the experimental results for both before (as-built) and after-cutting situations. There is a good agreement between the experimental and simulated results. The application of the local-global approach enables the reduction to a complex thermo-mechanical problem to a simpler structural-mechanical one. Using this approach, the required calculation time can be drastically reduced in two or more orders of magnitude. It can be concluded that the proposed method has good performance in simulation accuracy and efficiency.

\section{REFERENCES}

[1] Herzog D., Seyda V., Wycisk E., Emmelmann C. Additive manufacturing of metals. Acta. Mater (2016) 117:371-392.

[2] Teng, C. Gong, H. Szabo, A. Dilip, J.J.S. Ashby, K. Zhang, S. Patil, N. Pal, D. Stucker, B. Simulating melt pool shape and lack of fusion porosity for selective laser melting of cobalt chromium components. J. Manuf. Sci. Eng (2016) 139:11009.

[3] Buchbinder, D. Meiners, W. Pirch, N. Wissenbach, K. Schrage, J. Investigation on reducing distortion by preheating during manufacture of aluminum components using selective laser melting. J. Laser Appl (2014) 26:12004.

[4] Kempen, K. Vrancken, B. Buls, S. Thijs, L. Van Humbeeck, J. Kruth J.-P. Selective laser melting of crack-free high density M2 high speed steel parts by baseplate preheating. J. Manuf. Sci. Eng (2014) 136(6):061026.

[5] A. Keshavarzkermani, E. Marzbanrada, R. Esmaeilizadeha, Y.Mahmoodkhania, U. Ali, P. Enriquea, N.Y. Zhou, A. Bonakdar, E. Toyserkani, An investigation into the effect of process parameters on melt pool geometry, cell spacing, and grain refinement during laser powder bed fusion. Opt. Laser. Technol (2019) 116:83-91.

[6] Li, E.L. Wang, L. Yu, A.B. Zhou Z.Y. A three-phase model for simulation of heat transfer and melt pool behaviour in laser powder bed fusion process. J. Powder. Technol (2021) 381:298-312.

[7] Khairallah, S.A. Anderson, A.T. Rubenchik, A. King W.E. Laser powder-bed fusion additive manufacturing: Physics of complex melt flow and formation mechanisms of pores, spatter, and denudation zones. Acta. Materialia (2016) 108:36-45. 
[8] Russell, M. Souto-Iglesias, A. Zohdi, T. Numerical simulation of Laser Fusion Additive Manufacturing processes using the SPH method. Comput. Methods. Appl. Mech. Eng (2018) 341: 163-187.

[9] Fürstenau, J.-P. Wessels, H. Weißenfels, C., Wriggers, P. Generating virtual process maps of SLM using powder-scale SPH simulations. Comput. Part. Mech (2019) doi:10.1007/s40571-019-002963.

[10] Denlinger, E. R. Gouge, M. Irwin, J. Michaleris, P. Thermomechanical model development and in situ experimental validation of the Laser Powder-Bed Fusion process. Add. Manuf (2017) 16:7380 .

[11] Foroozmehr, A. Badrossamay, M. Foroozmehr, E. Golabi, S. Finite element simulation of selective laser melting process considering optical penetration depth of laser in powder bed. Mater. Des (2016) 89:255-263.

[12] Trappa, J., Rubenchikb, A.M. Gussb, G. Matthewsb, M.J. In situ absorptivity measurements of metallic powders during laser powder-bedfusion additive manufacturing. App. Mater. Today (2017) 9:341-349.

[13] Perzyna, P. Fundamental problems in viscoplasticity. Adv. Appl. Mech (1966) 9:243-377.

[14] Perzyna, P. Themodynamic Theory of Viscoplasticity. Adv. Appl. Mech (1971) 9:315-354.

[15] Owen, D.R.J. and Hinton, E. Finite element in plasticity: Theory and Practice. Pineridge Press Limited. Swansea. U.K. (1980).

[16] Inoue, T. and Nagaki, S. A. Constitutive Modeling of Thermoviscoelastic-Plastic Materials. $J$. Therm. Stresses (1978) 1:53-61.

[17] Inoue, T. Thermal stress III, Edited by R.B. Hetnarski, Elsevier Science Publishers B.V. (1989).

[18] Johnson, G. and Cook, W. Fracture characteristics of three metals subjected to various strains, strain rates, temperatures and pressures. J. Eng. Fracture. Mech (1985) 21:31-48.

[19] Wang, Z. and Inoue, T. Viscoplastic Constitutive Relation Incorporating Phase TransformationApplication to Welding. Mater. Sci. Techno (1985) 1:899-903.

[20] Cormeau, I. Numerical Stability in Quasi-Static Elasto/Visco-Plasticity. Int. J. Numer. Meth. Eng (1975) 9:109-127.

[21] Souloumiac, B. Boitout, F. and Bergheau, J.M. A new local-global approach for the modelling of welded steel component distortions. Math. Model. Weld. Phenom (2002) 6:573-590.

[22] Zhang, Z. Ibhadode, O. Ali, U. Dibia, C.F. Rahnama, P. Bonakdar, A. and Toyserkani, E. Topology optimization parallel-computing framework based on the inherent strain method for support structure design in laser powder-bed fusion additive manufacturing. Int. J. Mech. Mater. Des (2020) 16:897-923.

[23] Chen, Q., Liang, X., Hayduke, D., Liu, J., Cheng, L., Oskin, J., Ryan Whitmore, R. and To, A.C. An inherent strain based multiscale modeling framework for simulating part-scale residual deformation for direct metal laser sintering. Addit. Manuf (2019) 28:406-418.

[24] Denlinger, E.R., Gouge, M, Irwin J., Michaleris, P. Thermomechanical model development and in situ experimental validation of the Laser Powder-Bed Fusion process. Addit. Manuf(2017) 16:7380 .

[25] Cheng, B., Shrestha, S., Chou, K. Stress and deformation evaluations of scanning strategy effect in selective laser melting. Addit. Manuf (2016) 12:240-251. 